\title{
INDICADORES DE DESENVOLVIMENTO TECNOLÓGICO DE EMPRESAS NO SETOR INDUSTRIAL
}

\author{
Indicators of technological development of \\ corporations in industrial sector
}

Envio 21.04.08 / Aceite 29.07.08

\author{
Maria Aparecida Gouvêa ${ }^{1}$ \\ Vicente Lentini Plantullo²
}

\begin{abstract}
Resumo
O principal objetivo deste artigo foi captar, por meio de uma pesquisa qualitativa e uma pesquisa quantitativa exploratória, os recursos dentro de uma plataforma tecnológica que são atualmente mais utilizados por algumas empresas do setor industrial. Além disso, pretendeuse criar um indicador de desenvolvimento tecnológico para tais empresas. A técnica de análise fatorial foi aplicada a aspectos que refletem as declarações dos entrevistados a respeito da intensidade de utilização de mecanismos no contexto tecnológico em suas empresas. Foram, então, criados escores fatoriais por empresa, a partir dos quais se obteve uma medida sintética referente ao seu desenvolvimento tecnológico. Assim, foi possível obter um ranking com a posição relativa de cada empresa atuante no setor industrial.
\end{abstract}

Palavras-chave: Grau Tecnológico; Plataforma Tecnológica; Empresas Industriais; Análise Multivariada; Análise Fatorial.

\section{Abstract}

The main objective of this paper was to capture, by means of a qualitative investigation and an exploratory quantitative survey, the resources available on a technological platform

\footnotetext{
${ }^{1}$ Professora Livre-docente em Administração - FEA/USP; Professora no Departamento de Administração - FEA/USP; Editora da Revista REGE-USP (Revista de Gestão USP); Av. Prof. Luciano Gualberto, 908 - Cidade Universitária, sala E110 - Cep: 05508-900 - SP. Tel: (11) 3091 6044. Fax: (11) 38184039. E-mail: magouvea@usp.br

${ }^{2}$ Professor Pós-doutor - FEA/USP; Professor na Pontifícia Universidade Católica - PUC/SP. Av. Prof. Luciano Gualberto, 908 - Cidade Universitária, sala E110 - Cep: 05508-900 - SP. Tel: (11) 3091 6044. Fax: (11) 38184039. E-mail: vplentini@uol.com.br
} 
currently in widespread use by certain industrial corporations. Besides, it was intended to create a technological development indicator for these companies. The factor analysis technique was applied along with aspects that reflect the answers from the interviewees regarding the intensity of use of mechanisms in technological context by their companies. Then factor scores have been created to each company through which a summary measure of technological development was obtained in each company. Thus, it was possible to get a ranking with relative position of each company in industrial sector.

Keywords: Technological Degree; Technological Platform; Industrial Corporations; Multivariate Analysis; Factor Analysis.

\section{Introdução}

Tradicionalmente, a literatura não tem sido pródiga na produção de textos que contemplem o grau tecnológico das empresas (PLANTULLO, 2006). Pelo que se tem observado, os textos pertinentes ao grau tecnológico não são conclusivos, vez por outra se confundindo com os próprios termos técnicos do conceito. Não é raro encontrar textos com o uso sem distinção de termos, tais como: ciência, tecnologia, plataforma tecnológica, nível de implementação de software, hardware e outros. Há, ainda, o problema de se esperar avanço tecnológico muito acima da média com a compra de sofisticados equipamentos, sem o necessário treinamento e desenvolvimento de profissionais gabaritados.

Assim, percebeu-se a necessidade de mensuração do nível de desenvolvimento tecnológico das empresas, uma vez que, no entender de acadêmicos e profissionais de porte, seria possível utilizar conceitos estatísticos, de sorte a melhor esclarecer as ligações entre os níveis de desenvolvimento tecnológico das empresas, abrangendo o respeito às características individuais de cada funcionário, o ambiente de trabalho e o relacionamento entre pares, chefes, superiores e subordinados, tudo isso para maximizar o resultado empresarial para a comunidade como um todo (HOPP, 1995).

Assim sendo, o desenvolvimento deste trabalho pretende responder às indagações: industriais? e

- que variáveis são mais utilizadas para o desenvolvimento tecnológico de empresas tecnológico?

- qual posição cada empresa ocupa no ranking de um indicador de desenvolvimento

\section{Objetivo}

O objetivo central deste artigo é a apresentação dos recursos dentro de uma plataforma tecnológica que são atualmente mais utilizados por algumas empresas do setor industrial e que reflitam o seu grau tecnológico.

\section{Fundamentação teórica}

Cabe esclarecer aqui as diferenças entre ciência e tecnologia. Por ciência, entendese o saber, embora nem todo conhecimento seja considerado científico. O conhecimento científico passa por um processo metódico e sistemático de elaboração, de tal forma que suas constatações e proposições possam ser aceitas como verdadeiras. A ciência pode ser entendida 
como conjunto de conhecimentos científicos ou como a atividade sistemática e ordenada de forma a obter esses conhecimentos.

Longo (1979, p. 18-19) define ciência como "o conjunto organizado de conhecimentos relativos ao universo objetivo, envolvendo seus fenômenos naturais, ambientais e comportamentais". Já para Sábato (apud BARBIERI, 1983, p. 27), "é a atividade humana que tem por objetivo a busca dos conhecimentos da natureza mediante a aplicação do conjunto de regras que constituem o método científico".

Isso posto, a ciência pode ser básica (pesquisa pura e/ou fundamental) ou aplicada, conforme um dos inúmeros critérios de classificação. Ambas se valem do método científico; porém, na ciência ou pesquisa básica, a procura do conhecimento não está vinculada a objetivos práticos, como ocorre na ciência ou na pesquisa aplicada (RUSSELL, 1963). Conforme Bunge (1980, p. 28), enquanto na ciência básica o pesquisador estuda problemas do seu interesse, em função de motivos específicos; na ciência aplicada, ele estuda problemas de possíveis interesses sociais. Cabe destacar que o que ambas possuem em comum é o fato de terem por objetivo a busca dos conhecimentos que possam explicar a realidade e os métodos para sua obtenção.

Por tecnologia, entende-se o conhecimento das técnicas ou artes, compreendidas como habilidade e ofícios. A característica básica da tecnologia é ser essencialmente utilitária. Figueiredo (1972, p. 60) a define como a "soma de conhecimentos de natureza científica ou de natureza técnica, que se requerem para implantação e funcionamento de uma dada atividade industrial". O entendimento mais abrangente é fornecido por Longo (1979, p. 4): "tecnologia é o conjunto ordenado de todos os conhecimentos - científicos, empíricos ou intuitivos - empregados na produção e comercialização de bens e serviços". Sábato (apud BARBIERI, 1983, p. 5) observa que "o conjunto de conhecimentos propostos definidores de certa tecnologia deve estar ordenado, organizado e articulado".

A tecnologia, enquanto "conjunto ordenado, organizado e articulado de conhecimentos", pode estar ou não incorporada aos bens físicos ou tangíveis. No primeiro caso - tecnologia implícita ou incorporada -, esses conhecimentos materializam-se em bens físicos de capital e em insumos produtivos. Por outro lado, na tecnologia explícita ou nãoincorporada, o conjunto de conhecimentos está contido em documentos e nas pessoas pelas suas habilidades, experiências e capacitações profissionais.

Em suma, os recursos pertinentes à área de pesquisa e desenvolvimento de novos produtos, artigos, processos, serviços e informações devem ser subdivididos em alguns itens, a saber: recursos destinados à pesquisa pura, à pesquisa aplicada e ao efetivo desenvolvimento.

Os recursos destinados à pesquisa pura envolvem altas somas de numerário e se incluem no que é conhecido como sunk costs (HENDRICKSEN, 1977), sendo os prazos de maturação extremamente longos em virtude das incertezas reinantes no meio ambiente. A pesquisa pura é uma função, dentro da pesquisa e desenvolvimento (P \& D), que busca aplicações imediatas de conceitos, metodologias, filosofias e teorias desenvolvidas em nível global e que devem ser adaptadas à empresa capitalista industrial. Contudo, em grande parte das empresas industriais, comerciais, prestadoras de serviços e de informações, ela ainda deixa a desejar (ROUSSEL et al., 1992).

Com relação à pesquisa aplicada, é necessário o dispêndio de uma soma considerável de numerário, mas seu prazo de aplicação e maturação mostra-se sensivelmente menor, o que viabiliza as aplicações de cunho industrial.

Quanto à função desenvolvimento, é preciso entender que sua aplicação se dá nos inputs dos processos de fabricação, com o auxílio de engenharia, manutenção de máquinas e equipamentos, compras e suprimentos, controle da qualidade dentro e fora das linhas, e 
treinamento e desenvolvimento. Nessa função, inicia-se a produção de pequenos lotes, com vistas aos testes finais de introdução nos mercados globalizados (SUZAKI, 1993). Os resultados são quantificáveis no curto prazo e, essencialmente, práticos. O relacionamento dessa função com as demais se revela discreto, mas muito abaixo do desejado para a otimização dos processos.

Diversos trabalhos apresentam aspectos ligados à tecnologia e ao planejamento de desenvolvimento tecnológico nas empresas. À luz dessas publicações, algumas variáveis foram selecionadas para identificação do desenvolvimento tecnológico em empresas (na seção 4, é apresentado um quadro com tais variáveis).

Para a seleção de variáveis sobre grau tecnológico, foram focalizados alguns aspectos encontrados nas obras de autores que se dedicaram ao tema voltado à tecnologia. O Quadro 1 resume os aspectos e os respectivos autores.

\begin{tabular}{|l|l|}
\hline \multicolumn{1}{|c|}{ Aspectos } & \multicolumn{1}{c|}{ Autores } \\
\hline $\begin{array}{l}\text { A - Tecnologia de } \\
\text { Informação Inteligente }\end{array}$ & $\begin{array}{l}\text { Castelltort (1988), Scheer (1993), Rich e Knight (1994), } \\
\text { Meirelles (1995), Kovács (1996), Prigogine (1996), Krajewsky e } \\
\text { Ritzman (1999), Nussenzveig (2003) e Plantullo (2006). }\end{array}$ \\
\hline $\begin{array}{l}\text { B - Metodologias de } \\
\text { Froduço }\end{array}$ & $\begin{array}{l}\text { (1988), Osada (1989), Taguchi et al. (1990), Suzaki (1993) e } \\
\text { Deming (1990). }\end{array}$ \\
\hline Finanças - Metodologias de & Kaplan e Johnson (1987) e Cogan (2006). \\
\hline D - Filosofias Gerenciais & $\begin{array}{l}\text { Tomasko (1993), Hammer e Champy (1994), Morgan (1996) e } \\
\text { Santos (2005). }\end{array}$ \\
\hline E - Nível de Integração & Ansoff (2001) e Hamel e Prahalad (2005). \\
\hline F - Planejamento & $\begin{array}{l}\text { Roussel et al. (1992), Porter (2001), Ansoff (2001) e Hamel e } \\
\text { Prahalad (2005). }\end{array}$ \\
\hline
\end{tabular}

Quadro 1 - Aspectos sobre tecnologia.

\section{Aspectos metodológicos}

Para o enfoque simultâneo de variáveis correspondentes ao desenvolvimento tecnológico, foram pesquisadas várias publicações, chegando-se aos aspectos apresentados no Quadro 2, a seguir. 


\begin{tabular}{|c|c|}
\hline Variáveis & Suporte teórico - Aspectos do Quadro 1 \\
\hline 1. Atividades de Pequenos Grupos (APG's) & Q1B \\
\hline 2. Círculos de Controle da Qualidade (CCQ's) & Q1B \\
\hline 3. Computer - Aided Design (CAD) & Q1A \\
\hline 4. Computer - Aided Engineering (CAE) & Q1A \\
\hline 5. Computer - Aided Information Technology (CAIT) & Q1A \\
\hline 6.Computer - Aided Instruction (CAl) & Q1A \\
\hline 7. Computer - Aided Manufacturing (CAM) & Q1A \\
\hline 8.Computer - Aided Process Planning (CAPP) & Q1A \\
\hline 9. Computer - Aided Technology (CAT) & Q1A \\
\hline 10. Computer - Aided Testing (CAT) & Q1A \\
\hline 11. Computer - Integrated Manufacturing (CIM) & Q1A \\
\hline 12. Connectivity Among Systems (CAS) & Q1A \\
\hline 13. Controle Estatístico da Qualidade (CEQ) & Q1B \\
\hline 14. Controle Total da Qualidade (CTQ) & Q1B \\
\hline 15. Custeio Baseado em Atividade (CBA) & Q1C \\
\hline 16. Downsizing (DW) & Q1D \\
\hline $\begin{array}{l}\text { 17. Estratégia de Processos ou Manufatura } \\
\text { Integrados (EPMI) }\end{array}$ & Q1F \\
\hline $\begin{array}{l}\text { 18. Estratégia da Tecnologia da Informação } \\
\text { Integrada (ETII) }\end{array}$ & Q1F \\
\hline 19. Funções de Taguchi (FT) & Q1B \\
\hline 20. Gerenciamento Baseado na Atividade (GBA) & Q1C \\
\hline 21. Gerenciamento Total da Qualidade (GTQ) & Q1B \\
\hline 22. Global Sourcing (CS) & Q1B \\
\hline 23. Just-in-Case (IIC) & Q1B \\
\hline 24. Just-in-Time (IIT) & Q1B \\
\hline 25. Manutenção Produtiva Total (MPT) & Q1B \\
\hline $\begin{array}{l}\text { 26. Mudanças Organizacionais - Organizações } \\
\text { Transversais (MO-OT's) }\end{array}$ & Q1D, Q1F \\
\hline $\begin{array}{l}\text { 27. Organizações em Redes de Conhecimento } \\
\text { (ORC's) }\end{array}$ & Q1D \\
\hline
\end{tabular}

Quadro 2 - Variáveis sobre desenvolvimento tecnológico. 
O Quadro 3 expõe as fases do planejamento e desenvolvimento do estudo.

\begin{tabular}{|l|l|}
\hline \multicolumn{1}{|c|}{ Fases } & \multicolumn{1}{c|}{ Resultados } \\
\hline 1. Revisão bibliográfica & Maior embasamento teórico sobre o tema do trabalho. \\
\hline $\begin{array}{l}\text { 2. Elaboração do questionário- } \\
\text { piloto }\end{array}$ & $\begin{array}{l}\text { Detecção de ajustes necessários para aprimoramento do } \\
\text { instrumento de coleta. }\end{array}$ \\
\hline 3. Pesquisa qualitativa & $\begin{array}{l}\text { Realizada com dez executivos de empresas, sendo quatro } \\
\text { de empresas nacionais e seis de multinacionais. Os } \\
\text { respondentes procuraram fornecer sugestões, críticas, } \\
\text { contribuições e visões. }\end{array}$ \\
\hline 4. Pesquisa quantitativa & $\begin{array}{l}\text { Realizada com cento e sessenta e cinco executivos de } \\
\text { empresas nacionais e multinacionais. }\end{array}$ \\
\hline 5. Análise dos resultados & \begin{tabular}{l} 
Composta por cinco etapas, apresentadas na seção 5. \\
\hline
\end{tabular}
\end{tabular}

Quadro 3 - Fases do estudo.

Na primeira fase desta pesquisa, procedeu-se à leitura de livros, periódicos nacionais e internacionais, trabalhos de especialização, monografias de mestrado, teses de doutorado e de livre-docência, no sentido de formar o arquétipo teórico pertinente ao grau tecnológico e ao problema do nível de desempenho de empresas nacionais e/ou multinacionais. Em uma segunda fase, construiu-se um questionário-piloto com perguntas sobre o perfil das empresas e um grande bloco com as variáveis apresentadas no Quadro 2. Elaboraram-se questões fechadas, com 42 variáveis acerca da mensuração do grau tecnológico. Em uma terceira fase, aplicouse esse questionário-piloto para dez executivos, sendo quatro de empresas nacionais e seis de empresas multinacionais, para se obter um refinamento das questões na versão definitiva do questionário. Na quarta fase, realizou-se uma pesquisa de cunho exploratório, com 165 executivos e funcionários em nível de supervisão de empresas nacionais e multinacionais de diversos setores da economia, destacando-se que, em média, cada entrevista teve a duração de, aproximadamente, três a quatro horas, razão pela qual se justifica o tempo total gasto nesse processo - praticamente dois anos, de julho de 2004 a julho de 2006. A versão final do questionário constitui-se de três partes:

- parte A: dados gerais sobre a empresa;

- parte B: questões acerca das características do processo de produção da empresa;

- parte C: questões objetivas acerca do grau de desenvolvimento tecnológico da empresa.

$\mathrm{Na}$ quinta fase, concatenou-se todo o arcabouço teórico com a parte prática desenvolvida, aplicando-se algumas técnicas estatísticas.

A população focalizada neste estudo refere-se às empresas mencionadas na Revista Exame Melhores e Maiores, como as 500 maiores empresas do Brasil, no período de julho de 1994 a julho de 2006 (SANTOS e CARVALHO, 2006). Também se focalizaram algumas pequenas empresas, de sorte a se obter um possível contraste em termos de desempenho tecnológico. Pesquisaram-se 23 empresas, tendo mais de um respondente em cada uma. No total, entrevistaram-se 165 executivos. As empresas pesquisadas são as seguintes: 3M, Alcoa, 
Bunge, Colgate-Palmolive, Daimler-Chrysler, DASA, Engemix, Festo, Galvanoplastia Mauá Lanxess, Magneti Marelli-Cofap, Makita, Microsiga, Multibrás, Pichinin, Romi, Sanches-Blanes, Semasa, Siemens, Sig-Simonazzi, Usimac, Votoran Cimentos e Votorantim Metais.

\section{Análise dos resultados}

Nesta seção, são analisados os resultados em cinco etapas: missing values, outliers, estatísticas descritivas, análise fatorial e determinação do grau tecnológico.

\subsection{Análise dos missing values}

A freqüência de dados perdidos ou missing values é, em geral, da ordem de 10\%. Há algumas variáveis a serem eliminadas, devido ao fato de que possuem uma taxa de missing superior a $10 \%$, número considerado excessivo pela prática cotidiana, muito embora alguns autores não façam menção a um determinado percentual, ou seja, cada caso deve ser tratado de forma diferenciada (HAIR JUNIOR et al., 2005, p. 50-51, 58-60). Foram eliminadas as variáveis: $2,15, \mathrm{C} 16,19,20,23,26$ a 28, 33, 36 a 42. Além disso, as variáveis de números 1 , 12, 16, 24, 26 a 28 não representam fortemente o Grau Tecnológico das empresas pesquisadas, tendo, inclusive, muita sinergia com a área de Talentos e de Competências Humanas Essenciais (TCHE), antigo processo de Recursos Humanos (RH).

\subsection{Análise dos outliers}

Segundo Hair Junior et al. (2005, p. 72-73), faz-se mister que se verifique a presença ou não dos chamados pontos fora de observação ou outliers. Assim sendo, é importante o cálculo da distância de cada observação em relação a algum ponto comum. Para isso, necessita-se obter a chamada Distância de Mahalanobis em um espaço multidimensional de cada observação em relação ao centro médio das observações, o que forneceria uma mensuração comum de centralidade multidimensional. Hair Junior et al. (2005) sugerem um nível de 0,001 ou 0,1\% para designação de uma observação atípica.

A identificação de outliers foi feita para as variáveis mantidas após a análise dos missings.

Fazendo-se os cálculos da Distância $\mathrm{D}^{2}$ de Mahalanobis em relação ao número de graus de liberdade de todas as variáveis (no caso, 23), percebeu-se que o maior valor foi de 2,820 , sendo que o valor crítico é 3,768, ou seja, o maior valor é menor do que o valor crítico. Este resultado evidencia que não existem outliers nas respostas ofertadas pelas variáveis deste grupo, sinalizando, de certa forma, a estabilidade do estudo.

\subsection{Estatísticas descritivas}

Nesta seção, estudaram-se as principais estatísticas descritivas das variáveis mantidas após a análise dos missing values. Foram elas: Média, Mediana, Moda, Desvio-Padrão, Variância, Coeficiente de Variação, Assimetria, Curtose, Amplitude, Mínimo e Máximo Valor da Variável. A Tabela 1 apresenta estas estatísticas para as variáveis. 
Tabela 1 - Estatísticas descritivas

\begin{tabular}{|c|c|c|c|c|c|c|c|c|c|c|c|}
\hline VAR & MED & MD & MOD & DEP & VAR & $\mathrm{CV}$ & ASS & CUR & AMP & VMI & VMA \\
\hline 3 & 5,44 & 6 & 6 & 1,66 & 2,75 & 30,5 & $-1,35$ & 1,10 & 6 & 1 & 7 \\
\hline 4 & 5,19 & 6 & 7 & 1,84 & 3,40 & 35,5 & $-0,96$ & $-0,03$ & 6 & 1 & 7 \\
\hline 5 & 4,79 & 5 & 7 & 1,90 & 3,60 & 39,7 & $-0,52$ & $-0,86$ & 6 & 1 & 7 \\
\hline 6 & 4,66 & 5 & 6 & 1,84 & 3,40 & 39,5 & $-0,48$ & $-0,98$ & 6 & 1 & 7 \\
\hline 7 & 5,03 & 6 & 6 & 1,89 & 3,57 & 37,6 & $-1,04$ & $-0,07$ & 6 & 1 & 7 \\
\hline 8 & 5,21 & 6 & 6 & 1,85 & 3,42 & 35,5 & $-1,00$ & $-0,14$ & 6 & 1 & 7 \\
\hline 9 & 4,73 & 5 & 6 & 1,84 & 3,38 & 38,9 & $-0,90$ & $-0,27$ & 6 & 1 & 7 \\
\hline 10 & 4,65 & 5 & 6 & 1,89 & 3,56 & 40,6 & $-0,70$ & $-0,68$ & 6 & 1 & 7 \\
\hline 11 & 4,75 & 6 & 6 & 2,04 & 4,16 & 42,9 & $-0,75$ & $-0,78$ & 6 & 1 & 7 \\
\hline 13 & 5,45 & 6 & 6 & 1,47 & 2,17 & 27,0 & $-1,08$ & 0,71 & 6 & 1 & 7 \\
\hline 14 & 5,52 & 6 & 7 & 1,50 & 2,24 & 27,2 & $-1,03$ & 0,63 & 6 & 1 & 7 \\
\hline 17 & 4,8 & 5 & 6 & 1,55 & 2,39 & 32,3 & $-0,65$ & $-0,29$ & 6 & 1 & 7 \\
\hline 18 & 4,92 & 5 & 6 & 1,62 & 2,63 & 32,9 & $-0,90$ & 0,10 & 6 & 1 & 7 \\
\hline 21 & 5,32 & 6 & 6 & 1,50 & 2,24 & 28,2 & $-1,07$ & 0,62 & 6 & 1 & 7 \\
\hline 22 & 5,11 & 6 & 6 & 1,69 & 2,86 & 33,1 & $-1,04$ & 0,34 & 6 & 1 & 7 \\
\hline 25 & 5,15 & 5 & 6 & 1,46 & 2,13 & 28,3 & $-0,84$ & 0,06 & 6 & 1 & 7 \\
\hline 29 & 4,33 & 5 & 6 & 2,01 & 4,05 & 46,4 & $-0,31$ & $-1,15$ & 6 & 1 & 7 \\
\hline 30 & 4,83 & 5 & 6 & 1,89 & 3,58 & 39,1 & $-0,67$ & $-0,67$ & 6 & 1 & 7 \\
\hline 31 & 4,34 & 4 & 6 & 1,74 & 3,01 & 40,1 & $-0,32$ & $-0,80$ & 6 & 1 & 7 \\
\hline 32 & 4,11 & 4 & 5 & 1,55 & 2,42 & 37,7 & $-0,5$ & $-0,60$ & 6 & 1 & 7 \\
\hline 34 & 4,23 & 4 & 4 & 1,78 & 3,18 & 42,1 & $-0,16$ & $-0,95$ & 6 & 1 & 7 \\
\hline 35 & 4,32 & 4 & 6 & 1,64 & 2,68 & 38,0 & $-0,29$ & $-0,84$ & 6 & 1 & 7 \\
\hline
\end{tabular}

MED: Média, MD: Mediana, MOD: Moda, DEP: Desvio-Padrão, VAR: Variância, CV: Coeficiente de Variação, ASS: Assimetria, CUR: Curtose, AMP: Amplitude, VMI: Valor Mínimo e VMA: Valor Máximo. 
Cabe destacar que os valores dos coeficientes de variação em termos percentuais variaram de $27 \%$ (variável 13) para 63,9\% (variável 39). Esses resultados evidenciam que há uma miríade de respostas diferentes entre si. Além disso, percebeu-se que a variável 14 apresentou o maior valor médio: 5,52. Por outro lado, as variáveis 3, 4, 7, 8, 11, 13, 14, 21 e 22 apresentaram os maiores valores da mediana, enquanto os maiores valores da moda foram encontrados nas seguintes variáveis: 4, 5 e 14 .

\subsection{Análise fatorial}

Quando se trabalha com dados de variáveis para serem devidamente analisados por meio da Análise Fatorial (AF), Hair Junior et al. (2005, p. 94) ensinam que existem números primordiais para a execução desta técnica. Assim sendo, neste caso prático, pode-se perceber que a relação matemática entre o número de observações válidas pelo número total de variáveis envolvidas é de 104/23, o que fornece o número de 4,52, muito próximo de 5,00. O ideal é que fossem dez observações (HAIR JUNIOR et al., 2005, p. 98) por variável, mas isso raramente se reproduz na prática, sobretudo em uma pesquisa exploratória, envolvendo a obtenção de dados primários por meio de entrevistas com gerentes e diretores, quer de empresas nacionais, quer de multinacionais/transnacionais.

A hipótese básica desta técnica repousa na existência de correlações entre as variáveis, cuja causa decorra de fatores comuns compartilhados entre elas. O que se pratica, com esta técnica, é a análise da interdependência entre as variáveis, a partir da matriz de correlação. Esta técnica possibilita a captação de fatores não diretamente observáveis, a partir das variáveis conhecidas pelo levantamento dos dados de campo. Na análise do fator comum, as variáveis são agrupadas em função de suas correlações, significando que variáveis que compõem um determinado fator devem ser altamente correlacionadas entre si e fracamente correlacionadas com as variáveis que entram na composição do outro fator.

A matriz de correlação sinalizou a existência de correlações significantes entre pares de variáveis, justificando-se a premissa de que, de fato, estas compartilham fatores em comum. Este fato reforça a premissa de existência de fatores compartilhados pelas variáveis e a pertinência de se aplicar a análise fatorial.

A Tabela 2 apresenta a medida KMO e o teste de Bartlett.

Tabela 2 - Medida KMO e teste de Bartlett

Mensuração de adequação da amostra segundo os critérios de
Kaiser-Meyer-Olkin (KMO)

A medida KMO (estatística que confronta as correlações entre as variáveis com as correlações residuais) mostrou-se adequada, pois seu valor é alto e próximo de 1,0. Como esperado, a hipótese formulada no teste de Bartlett (similaridade da matriz de correlação com a matriz identidade) foi rejeitada, endossando a existência de fatores subjacentes às variáveis. 
Quanto à comunalidade (porcentagem da variância de cada variável explicada pelos fatores obtidos na análise fatorial), os resultados são satisfatórios. O maior e menor resultado refere-se às variáveis $30(0,805)$ e $25(0,591)$.

Foram obtidos 4, que captaram $71,2 \%$ do total da variância das variáveis.

As cargas fatoriais refletem a importância dos fatores na composição de cada variável e, reciprocamente, o peso das variáveis em cada fator.

A Tabela 3 apresenta a alocação das variáveis nos fatores, segundo as cargas fatoriais.

Tabela 3 - Matriz dos fatores

\begin{tabular}{|c|c|c|c|c|}
\hline \multirow[t]{2}{*}{ Variáveis } & \multicolumn{4}{|c|}{ Fatores } \\
\hline & 1 & 2 & 3 & 4 \\
\hline 3 & 0,781 & 0,151 & 0,225 & $7,335 \mathrm{E}-02$ \\
\hline 4 & 0,821 & 0,204 & 0,137 & 0,219 \\
\hline 5 & 0,685 & 0,173 & $-0,127$ & 0,467 \\
\hline 6 & 0,595 & 0,260 & $-0,014$ & 0,442 \\
\hline 7 & 0,802 & 0,232 & 0,234 & 0,214 \\
\hline 8 & 0,817 & 0,319 & 0,163 & $3,219 \mathrm{E}-02$ \\
\hline 9 & 0,817 & 0,214 & 0,102 & 0,165 \\
\hline 10 & 0,803 & 0,297 & 0,142 & 0,155 \\
\hline 11 & 0,705 & 0,240 & 0,253 & 0,382 \\
\hline 13 & 0,615 & 0,545 & 0,125 & 0,219 \\
\hline 14 & 0,491 & 0,588 & $9,765 \mathrm{E}-02$ & 0,220 \\
\hline 17 & 0,287 & $9,478 \mathrm{E}-02$ & 0,402 & 0,606 \\
\hline 18 & 0,418 & 0,601 & $2,950 \mathrm{E}-02$ & 0,290 \\
\hline 21 & 0,318 & 0,662 & 0,274 & $6,408 \mathrm{E}-02$ \\
\hline 22 & 0,612 & 0,460 & 0,203 & 0,165 \\
\hline 25 & 0,342 & 0,649 & 0,144 & 0,179 \\
\hline 29 & 0,217 & 0,285 & $2,065 \mathrm{E}-02$ & 0,811 \\
\hline 30 & $5,763 \mathrm{E}-02$ & 0,872 & $9,242 \mathrm{E}-02$ & 0,182 \\
\hline 31 & 0,222 & 0,560 & $-0,010$ & 0,607 \\
\hline
\end{tabular}


(continuação da Tabela 3)

\begin{tabular}{l|l|l|l|l}
\hline 32 & 0,325 & $\mathbf{0 , 5 3 6}$ & 0,508 & $5,196 \mathrm{E}-02$ \\
\hline 34 & 0,245 & $2,760 \mathrm{E}-02$ & $\mathbf{0 , 7 9 7}$ & 0,192 \\
\hline 35 & 0,400 & 0,172 & $\mathbf{0 , 6 7 0}$ & $-0,151$ \\
\hline 39 & $-0,102$ & 0,182 & $\mathbf{0 , 8 7 0}$ & $5,898 \mathrm{E}-02$ \\
\hline
\end{tabular}

Nomeação dos fatores:

a) Fator da Tecnologia da Informação Inteligente (FTII), abrangendo as variáveis 3 a 11, 13 e 22, uma vez que este fator detém componentes essencialmente computacionais embasados em tecnologia, informação, coleta, armazenagem de dados, transformação de dados em informações e, sobretudo, a ilação entre tecnologia, informação e telecomunicações, cerne da era digital em que está inserido. É um diferencial competitivo significativo;

b) Fator Estratégico e Produtivo Internos (FEPI), abrangendo as variáveis 14, 18, 21, 25, 30 e 32, uma vez que envolve posicionamentos estratégicos internos da organização, acoplados com métodos, ferramentas e metodologias de maximização da produtividade das máquinas e equipamentos como um todo;

c) Fator Pontual-Estrutural (FP-E), abrangendo as variáveis 34, 35 e 39, em que predomina a necessidade de se focar um determinado problema ou problemas com maestria, além de modificar a estrutura das empresas, tornando-as mais leves, ágeis, de sorte a enfrentar a concorrência globalizada;

d) Fator Estratégico Externo (FEE), abrangendo as variáveis 17, 29 e 31, não só no que tange ao planejamento estratégico tradicional proposto por Ansoff (2001) e outros, mas também pelo planejamento estratégico reverso, com mudança de paradigmas proposto por Hamel e Prahalad (2005).

\subsection{Determinação do grau tecnológico}

Nesta seção, pretende-se determinar o grau tecnológico de empresas industriais. Trata-se de um tema de complexa solução, uma vez que se devem analisar as proporções de capital fixo que as empresas possuem; se as máquinas e/ou equipamentos acompanham o desenvolvimento tecnológico mundial; se há uma relação entre o capital fixo e o capital total, aqui entendido como a soma do capital fixo mais o variável; se a empresa possui um nível de lançamento de vetores produto no mercado; se a empresa treina e desenvolve bem seus colaboradores, dentre outras considerações.

Como a maioria desses dados não foi passível de obtenção, passou-se a inquirir os principais executivos das empresas pesquisadas acerca dos itens pertinentes ao questionário deste trabalho exploratório.

Para o cálculo do Grau Tecnológico, aplicou-se a seguinte fórmula (FACHEL, 1976, p. 76-77):

$$
\mathrm{I}_{\mathrm{i}, \mathrm{c}}=\sum_{\mathrm{j}=1}^{4} \mathrm{~F}_{\mathrm{ij}, \mathrm{C}} \cdot \mathrm{P}_{\mathrm{j}}
$$


em que:

- li,C = Índice Global do Grau Tecnológico da i-ésima empresa, i varia de 1 a 104;

- Fij,C = Escore fatorial para a i-ésima empresa e j-ésimo fator;

- Pj = Percentual de Variância Explicada pelo Fator j antes e depois da rotação, sem dividir por 100.

Foram calculadas as médias dos índices com base nos escores fatoriais. Em seguida, os dados foram padronizados, dividindo-se cada média pelo valor máximo observado. A média máxima obtida foi 37,96, referente à empresa Siemens. pesquisadas.

A Tabela 4 apresenta os índices de grau tecnológico calculados para as empresas

Tabela 4 - Grau tecnológico das empresas

\begin{tabular}{|c|c|}
\hline $3 M$ & 18,17 \\
\hline Alcoa & 6,22 \\
\hline Bunge & $-95,30$ \\
\hline Colgate-Palmolive & 79,37 \\
\hline Daimler-Chrysler & 43,91 \\
\hline Diagnósticos das Américas & 44,09 \\
\hline Engemix & $-303,08$ \\
\hline Festo & $-12,14$ \\
\hline Galvanoplastia Mauá & $-170,07$ \\
\hline Lanxess & 0,79 \\
\hline Magnetti-Marelli & $-12,18$ \\
\hline Makita & 0,00 \\
\hline Microsiga & $-99,92$ \\
\hline Multibrás & 60,37 \\
\hline Pichinin & 0,00 \\
\hline Romi & 27,71 \\
\hline Sanches-Blanes & $-176,38$ \\
\hline Siemens & 100,00 \\
\hline Sig-Simonazzi & $-6,07$ \\
\hline Usimac & 58,86 \\
\hline Votoran - Cimentos & $-118,96$ \\
\hline Votorantim Metais & 0,00 \\
\hline
\end{tabular}


Como se pode depreender da Tabela 4, as empresas Siemens, Colgate-Palmolive, Multibrás, Usimac, Diagnósticos das Américas e Daimler-Chrysler ocupam posições de destaque em termos de grau tecnológico. Assim, foi possível obter a posição relativa das empresas pesquisadas em termos de seu avanço no contexto tecnológico.

\section{Conclusão}

À luz do referencial teórico apresentado, selecionou-se as variáveis de interesse neste trabalho com o intuito de se aferir o grau tecnológico das empresas industriais. Além disso, pelos resultados apresentados, puderam-se destacar importantes informações, tais como:

a) as variáveis mais utilizadas para o desenvolvimento tecnológico de empresas industriais, segundo a média aritmética, são: 14. Controle Total da Qualidade (CTQ), 13. Controle Estatístico da Qualidade (CEQ), 3. Computer-Aided Design (CAD), 21. Gerenciamento Total da Qualidade (GTQ), 8. Computer - Aided Process Planning (CAPP), 4. Computer - Aided Engineering (CAE), 23. Just-in-Case (JIC), 22. Global Sourcing (GS) e 7. Computer - Aided Manufacturing (CAM);

b) este estudo sinalizou diferenças nos níveis de desempenho e nas ações empreendidas por algumas empresas do setor industrial em relação ao seu desenvolvimento tecnológico, o qual detém extrema importância em um cenário cada vez mais competitivo.

\section{Referências}

ANSOFF, H. I. A nova estratégia empresarial. São Paulo: Atlas, 2001.

BARBIERI, J. C. Incentivos à produção de tecnologia no Brasil. 1983, 152 páginas Dissertação (Mestrado em Administração) Curso de Pós-Graduação em Administração da Produção e Operações Industriais, da Escola de Administração de Empresas de São Paulo/ Fundação Getúlio Vargas, São Paulo, 1983.

BUNGE, M. Ciência e desenvolvimento. Belo Horizonte, Itatiaia e São Paulo: Universidade de São Paulo, 1980. (Coleção o Homem e a Ciência, 11).

CASTELLTORT, X. CAD CAM: metodologia e aplicações práticas. São Paulo: McGraw-Hill, 1988.

COGAN, S. Modelo de custeio baseado em atividades aplicado a decisões de produção de curto prazo. Contabilidade Vista \& Revista, v.17, p.29-46, 2006.
DEMING, W.E. Qualidade: a revolução da administração. Rio de Janeiro: Marques Saraiva, 1990.

FACHEL, J.M.G. Análise fatorial. Dissertação

(Mestrado em Estatística) - Instituto de Matemática e Estatística - Universidade de São Paulo, São Paulo, 1976.

FEIGENBAUM, A. V. Quality control. New York, USA: McGraw-Hill, 1951.

FIGUEIREDO, N. F. A transferência de tecnologia do desenvolvimento industrial do Brasil. Rio de Janeiro: IPEA, 1972.

GOLDRATT, E. M.; COX, J. A meta: um processo de melhoria contínua. São Paulo: Nobel, 1984.

HAIR JUNIOR, J. F. et al. Análise multivariada de dados. 5. ed. Porto Alegre: Artmed/Bookman, 2005. 593p.

HAMEL, G.; PRAHALAD, C. K. Strategic intent. Harvard Business Review Article, July 01, 2005. 
HAMMER, M.; CHAMPY, J. Reengenharia: revolucionando a empresa em função dos clientes, da concorrência e das grandes mudanças da gerência. 13. ed. Rio de Janeiro: Campus, 1994.

HENDRICKSEN, E. S. Accounting theory. United States: R. D. Irwin, 1977.

HOPP, J. C. Notas de aula das disciplinas Teoria Superior de Contabilidade e Contabilidade de Custos ministradas em nível de doutorado nas dependências da Escola de Administração de Empresas de São Paulo da Fundação Getúlio Vargas, 1995. S. n. t.

JURAN, J. M.; GRYNA, F. M. Juran's quality control handbook. 4. ed. Singapore: McGrawHill, 1988. (Industrial Quality Series).

\section{KAPLAN, R. S.; JOHNSON, T. H. Relevance}

lost: the rise and fall of management accounting. Massachusetts: Harvard School Business Press, 1987.

KOVÁCS, Z. L. Redes neurais artificiais fundamentos e aplicações: um texto básico. São Paulo: Edição Acadêmica, 1996

KRAJEWSKI, L. J.; RITZMAN, L. P. Operations management. Massachusetts: Addison Wesley, 1999.

LONGO, W. P. Informativo do INT. v. 3, n. 23, p.3-19. set./dez. 1979.

MEIRELLES, F. de S. Notas de aula: disciplina Administração de Recursos de Informática ministrada em nível de mestrado nas dependências da Escola de Administração de Empresas de São Paulo da Fundação Getúlio Vargas, 1995. S. n. t.

MORGAN, G. Imagens da organização. São Paulo: Atlas, 1996.

NUSSENZVEIG, H. M. (Org.). Complexidade \& caos. 2. ed. Rio de Janeiro: UFRJ/COPEA, 2003. 276p.

OSADA, T. Housekeeping: 5's: seiri, seiton, seiso, seiketsu, shitsuke. Cinco pontos-chave para o ambiente da qualidade. São Paulo: IMAM, 1989.
PLANTULLO, V. L. Um estudo empírico do grau tecnológico e dos modelos de treinamento e desenvolvimento de empresas industriais. 2006. 346 f. Tese (Pós-Doutorado em Administração de Empresas) - Faculdade de Economia, Administração e Contabilidade Universidade de São Paulo, São Paulo, 2006.

PORTER, M. Strategy and Internet. Harvard Business Review, p. 63-78, Mar. 2001.

PRIGOGINE, I. O fim das certezas: tempo, caos e as leis da natureza. São Paulo: Ed. da UNESP, 1996.

RICH, E.; KNIGHT, K. Inteligência artificial. 2.ed. São Paulo: Makron Books, 1994.

ROUSSEL, P. et al. Pesquisa \& desenvolvimento: como integrar P\&D ao plano estratégico e operacional das empresas como fator de produtividade e competitividade. São Paulo: Makron Books/Arthur D. Little, 1992.

RUSSELL, B. Knowledge by acquaintance and knowledge by description. Proceedings of the Aristotelian Society. London: Allen and Unwin, 1963.

SANTOS, A. D; CARVALHO, N. Revista Exame melhores e maiores: as 500 maiores empresas do Brasil. p.58-62 e p.68-87, jul. 2006.

\section{SANTOS, S. A. D. (Org.). Empreendedorismo} de base tecnológica: evolução e trajetória. 2 . ed. Maringá, Paraná: UNICORPORE Educação e Comunicação Corporativas, 2005.

SCHEER, A.-W. CIM: evoluindo para a fábrica do futuro. 2. ed. Rio de Janeiro: Qualitymark, 1993.

SUZAKI, K. The new shop floor management: empowering people for continuous improvement. New York, USA: The Free, 1993.

TAGUCHI, G. et al. Taguchi: engenharia da qualidade em sistemas de produção. São Paulo: Mc-Graw Hill, 1990.

TOMASKO, R. M. Rethinking: repensando as corporações. Reengenharia e gestão de mudanças. São Paulo: McGraw-Hill, 1993. 